\title{
Revista Internacional de Lingüística Iberoamericana (= RILI). Volumen XIV. No. 1 (27). 2016. Sección temática: La atenuación y la intensificación desde una perspectiva semántico-pragmática. Madrid/Frankfurt: Iberoamericana/Vervuert. 238 pp. ISSN 1579-9425 Reseña
}

\author{
GLORIA UCLÉS RAMADA ${ }^{1}$
}

1Universitat de Valéncia. Gloria.Ucles@uv.es

El número de la Revista Internacional de Lingüística Iberoamericana que reseñamos a continuación dedica una sección temática a la atenuación y la intensificación, coordinada por Wiltrud Mihatsch y Marta Albelda. Asimismo, también consta de una sección general con los artículos sobre marcadores discursivos de Portolés y sobre semántica de Bermúdez y de Van Gorp y Delbecque.

La sección temática se centra en la atenuación y la intensificación e incluye ocho artículos con un acercamiento eminentemente discursivo y de corpus. En gran parte de los estudios de este apartado, se sigue la propuesta teórica de Briz y Albelda (2013) y Albelda et al (2014) en las que distinguen tres funciones de la atenuación: la autoprotección, centrada en proteger posibles amenazas a la imagen del propio hablante; la prevención, que intenta evitar daños a la imagen del interlocutor, y por último la prevención, usada para reparar un daño lingüístico causado anteriormente. Los dos primeros artículos (la introducción de Mihatsch y Albelda y el trabajo de Marta Albelda) abordan cuestiones del nivel macro, relacionadas con aspectos teóricos de la atenuación. Los seis trabajos restantes se centran en estudios particulares, cinco de ellos enfocados a casos particulares de atenuación -expresada mediante distintos mecanismos lingüísticos y en distintos géneros discursivos(en concreto, los trabajos de Cabedo, Soler, Villalba, Kornfeld y Flores Treviño) y uno de ellos, en formas que basculan entre la intensificación y la atenuación o que pueden manifestar ambas (el de Lavric).

La introducción a la sección temática de Wiltrud Mihatsch y Marta Albelda aborda dos aspectos relevantes para el estudio de la atenuación y la intensificación. En primer lugar, definen el concepto de intensidad como la posición que ocupan distintos valores respecto a una escala con un punto de referencia que puede ser rebasado en dirección ascendente o descendente. Estas escalas pueden ser semánticas (de cuantificación, de dimensión, de 
grado de intensidad de una propiedad), pragmáticas (de fuerza ilocutiva y de grados de afectividad y expresividad) o semántico-pragmáticas (escalas de modalidad deóntica y epistémica). Según estas autoras, la diferencia entre atenuación e intensificación resulta más clara que las diferencias entre las distintas escalas de intensidad. En segundo lugar, discuten la relación que se establece entre atenuación e intensificación. Mihatsch y Albelda defiende que el hecho de que expresiones que típicamente se ligan a uno de estos dos fenómenos también pueda desarrollar ambos se debe, entre otros casos, bien a usos irónicos, a la naturaleza ambigua de las propias expresiones o a la aparición en determinadas construcciones.

En su artículo «Sobre la incidencia de la imagen en la atenuación pragmática», Marta Albelda se propone comprobar si la imagen está en la base de la noción de atenuación. Para ello, lleva a cabo una revisión de las definiciones teóricas de atenuación, y analiza trabajos propios y ajenos construidos sobre corpus. Su labor de comparación la lleva a afirmar que el concepto de imagen aparece en la mayoría de las descripciones teóricas de la atenuación, y además aporta pruebas de que son dudosos todos los casos de atenuación en los que originalmente no se había considerado que hubiera una imagen implicada. Como resultado de este doble trabajo teórico y práctico, la autora concluye que la imagen está necesariamente ligada a la atenuación.

Los estudios particulares sobre elementos atenuantes se abren con el artículo de Adrián Cabedo, quien en «La función de la atenuación y la configuración prosódica: un estudio a partir de un corpus de español coloquial» presenta un análisis exploratorio que pretende reivindicar el papel de la prosodia como elemento atenuador y establecer las variables fónicas para el reconocimiento de las distintas funciones de la atenuación, tal y como se describen en Albelda y Briz (2013). A partir de dos conversaciones coloquiales del corpus Val.Es.Co. 2.0 (Cabedo y Pons, 2013) se analizan las muestras de atenuación fónica y se tratan estadísticamente, de manera que se revelan determinadas tendencias: el primer resultado destacable es que la mitad de los casos analizados solo se producen por atenuación fónica, esto es, no están acompañados de ningún tipo de forma lingüística atenuante. Asimismo, la función de autoprotección parece asociarse a una frecuencia e intensidad bajas, mientras que la función de prevención suele manifestarse, además de con los rasgos anteriores -esto es, con baja frecuencia e intensidad-, con una fuerte modulación y alargamientos frecuentes. Por su parte, la función de reparación rompe la tendencia de las dos funciones anteriores y se asocia con una prominencia fónica elevada, con frecuencia e intensidad altas.

El valor atenuante de los verbos creer, pensar, opinar y suponer es el objeto de estudio de «La función atenuante en los verbos doxáticos del español», de Amparo Soler. La autora defiende que este tipo de verbos tienen dos lecturas, esto es, una lectura fuerte, en la que marcan adhesión del hablante con lo dicho, y una lectura débil, en la que su uso implica una suavización del compromiso con las palabras emitidas. A partir del análisis de muestras orales conversacionales y escritas periodísticas se establece que, en 
todos los casos de lectura débil, los verbos de opinión estudiados son atenuantes, mientras que la lectura fuerte puede asociarse tanto a atenuación como a intensificación, además de desarrollar otras funciones.

Cristina Villalba discute si en el género de los juicios orales, caracterizado por su rigidez, las fórmulas de tratamiento pueden adquirir una dimensión estratégica como elementos atenuantes. En «Las formas de tratamiento nominales y fórmulas apelativas convencionalizadas en los juicios orales: ¿elementos ritualizados o estrategias de atenuación?», la autora concluye, a partir de un análisis de un corpus, que estos elementos sí pueden tener una función atenuante, y que esta función se asocia a la voluntad de los participantes en el juicio -especialmente de los abogados- de preservar la armonía y evitar los conflictos durante las vistas. En cuanto a la función desempeñada por la atenuación (Briz y Albelda, 2013), señala que en los juicios se emplea fundamentalmente como estrategia de prevención de daños a la imagen ajena $y$, solo en menor medida, la de reparación de un daño ya infligido.

La relación entre locuciones indeterminadas y la atenuación es el objeto de estudio de Ana Llopis en «De la indeterminación a la atenuación: de alguna manera/forma/modo». De nuevo, mediante un análisis de un corpus que engloba seis géneros distintos (académico, ensayo, novela, prensa, entrevista y conversación) estudia las locuciones de alguna manera/forma/modo.

Estos marcadores se presentan con dos lecturas posibles: una lectura específica, en la que el emisor se refiere a un referente identificable, y una lectura no específica, en la que no se alude a un referente, y ambas lecturas pueden desarrollar valores atenuantes. En cuanto al tipo de atenuación que desarrollan estas formas, predomina un uso como protector de la propia imagen del hablante $y$, en menor medida, como estrategia preventiva de daños al oyente.

Los diminutivos son el tema central de «Una propuestita astutita: el diminutivo como recurso atenuador», de Laura Malena Kornfeld. El trabajo estudia la combinatoria del diminutivo -ito y atiende a los valores atenuantes que puede desarrollar. Según esta autora, la atenuación expresada por esta forma de diminutivo parece estar ligada con dos casos: en primer lugar, con combinaciones no esperables, como es el caso de los nombres de masa, dado que sus límites difusos no propician una caracterización como 'pequeño'. En segundo lugar, con adjetivos apreciativos que contienen una valoración negativa en su significado (rápido/ rapidito vs. veloz/*velocito) o que pueden adquirir connotaciones negativas en ciertos contextos determinados.

En el artículo «Correspondencia de la atenuación e intensificación en el uso de bastante y su derivación en el habla del noreste de México», María Eugenia Flores Treviño se vale de un corpus de entrevistas recopilado entre 1985-1986 en Monterrey y una muestra del subcorpus de PRESEEA correspondiente a esta ciudad mexicana. En ellos se estudian, más 
concretamente, los valores atenuantes de la forma bastantito sirviéndose de la ficha de atenuación propuesta por Albelda et álii (2014). Los datos apuntan a que bastantito, que en el plano semántico funciona como cuantificador, se utiliza como atenuante en la dimensión pragmática. Más concretamente tiene como cometido proteger la imagen del hablante, así como prevenir daños en la del oyente.

Un artículo, como se señalaba anteriormente, abordan la relación y las fronteras entre la categoría de atenuación y la categoría pragmática vecina, la intensificación. Eva Lavric atiende a la atenuación y la intensificación de los determinantes en el trabajo «iAy, Señor/ qué juventud esta! Atenuar e intensificar con determinantes (en un corpus escrito y otro conversacional)». La principal idea que subyace en el trabajo es que la mayoría de los casos que analiza -tanto si se trata de muestras del discurso escrito como del conversacional- son clasificados como casos de intensificación, mientras que la atenuación presenta resultados marginales.

La sección general de la revista consta de tres artículos: el primero se centra en la descripción de marcadores del discurso, mientras que los otros dos se inscriben dentro del campo de la semántica: concretamente uno trata la evidencialidad en los tiempos verbales y el otro, la descripción de verbos desde el cognitivismo.

En «Razón de más como inversor argumentativo», José Portolés estudia el funcionamiento de este marcador discursivo. Para ello se sirve del esquema

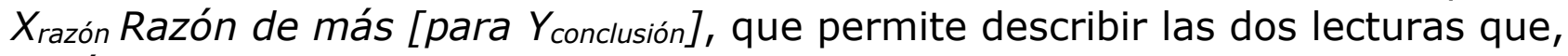
según este autor, puede desarrollar el marcador: por una parte, en contextos monologales, en razón de más la causa y la conclusión están coorientadas y, por tanto, el argumento $\mathrm{X}$ es la razón de $\mathrm{Y}$. Por otra parte, en casos dialogales, proponente y oponente invocan el mismo topos para llegar a conclusiones opuestas: precisamente la razón que da el proponente para defender una línea argumentativa es la que permite al oponente derivar la contraria.

Fernando Bermúdez se propone describir el uso modal y aspectual de los tiempos verbales en futuro en el castellano del Río de la Plata en el artículo «<ir a+infinitivo> Modalidad y aspecto en el futuro del castellano rioplatense». Su trabajo está basado en la distinción entre eventos comenzados y no comenzados como parte de la noción de aspecto. Así pues, relaciona la perífrasis ir a + infinitivo con la expresión de eventos no comenzados.

Aprovecha esta línea de trabajo para describir otras formas perifrásticas de futuro: ir a + infinitivo compuesto marca una acción no comenzada y terminada, irá a + infinitivo se emplea con acciones no comenzadas que expresan modalidad epistémica e iba a + infinitivo está ligado a eventos no comenzados y no terminados. Por otra parte, defiende que en el futuro simple se da la modalidad epistémica, pero no está presente la noción aspectual de evento terminado o no terminado. 
En el artículo que cierra el número de esta revista, Lise Van Gorp y Nicole Delbecque se centran en los valores modales verbo hacerse en «La dimensión subjetiva en el uso del verbo pseudocopulativo de cambio hacerse». A través de un análisis de corpus, las autoras sostienen que este verbo puede adquirir diferentes grados de subjetividad. Se pueden encontrar ejemplos transparentes donde el conceptualizador no interviene en el cambio que el atributo presenta sobre el sujeto. Por otra parte, ligado a atributos de tipo modal, evidencial o de percepción sensorial, catalogan los casos en los que el cambio tiene lugar en la perspectiva subjetiva del conceptualizador. Por último, se describen los casos que consideran de mayor subjetividad (denominados hipersubjetivos), en los que el cambio no ocurre en el mundo físico, sino que se produce en la mente del conceptualizador.

Consideramos que la sección especializada de este volumen es de consulta imprescindible para todos aquellos que estudien la modalización y, en especial, para quienes trabajen en atenuación e intensificación, ya que en ella participan expertos en esta materia. De hecho, sus aportaciones contribuyen a una mayor comprensión del funcionamiento de la atenuación e intensificación en diferentes manifestaciones lingüísticas y paralingüísticas. Muchos de los artículos que aquí se presentan constan asimismo de una base teórica y metodológica común: los estudios sobre la atenuación de Briz y Albelda (2013) y Albelda et al (2014). Este hecho prueba, por tanto, la aplicabilidad de la propuesta de Valencia y su consolidación como base de definición y análisis de la atenuación para otros trabajos dentro de este ámbito.

\section{Referencias bibliográficas}

Albelda Marco, Marta, Antonio Briz Gómez, Ana María Cestero, Dorota Kotwica y Cristina Villalba Ibáñez. 2014. Ficha metodológica para el análisis pragmático de la atenuación en corpus discursivos del español. (es.por.atenuación), Oralia, 17: 7-62.

Briz Gómez, Antonio y Marta Albelda Marco. 2013. Una propuesta teórica y metodológica de la atenuación lingüística en español y portugués. La base de un proyecto en común (es.por.atenuación), Onomázein, 28: 288-319.

Cabedo Nebot, Adrián y Salvador Pons Bordería. 2013. Corpus Val.Es.Co. 2.0. [en línea] Disponible en htpp://www.valesco.es 